Article

\title{
New Insights into the State Trapping of UV-Excited Thymine
}

\author{
Ljiljana Stojanović ${ }^{1}$, Shuming Bai ${ }^{1}$, Jayashree Nagesh ${ }^{2}$, Artur F. Izmaylov ${ }^{2,3}$, \\ Rachel Crespo-Otero ${ }^{4}$, Hans Lischka ${ }^{5,6}$ and Mario Barbatti ${ }^{1, *}$ \\ 1 Aix Marseille Univ., CNRS, ICR, Marseille, France; ljiljana.stojanovic@univ-amu.fr (L.S.); \\ shuming.bai@univ-amu.fr (S.B.) \\ 2 Chemical Physics Theory Group, Department of Chemistry, University of Toronto, \\ Toronto, ON M5S 3H6, Canada; jnagesh@chem.utoronto.ca (J.N.); artur.izmaylov@utoronto.ca (A.F.I.) \\ 3 Department of Physical and Environmental Sciences, University of Toronto Scarborough, \\ Toronto, ON M1C 1A4, Canada \\ 4 School of Biological and Chemical Sciences, Queen Mary University of London, Mile End Road, \\ London E1 4NS, UK; r.crespo-otero@qmul.ac.uk \\ 5 School of Pharmaceutical Sciences and Technology, Tianjin University, Tianjin 300072, China; \\ hans.lischka@univie.ac.at \\ 6 Department of Chemistry and Biochemistry, Texas Tech University, Lubbock, TX 79409, USA \\ * Correspondence: mario.barbatti@univ-amu.fr; Tel.: +33-4-8452-9200
}

Academic Editor: Carlos Crespo-Hernandez

Received: 12 October 2016; Accepted: 17 November 2016; Published: 23 November 2016

\begin{abstract}
After UV excitation, gas phase thymine returns to a ground state in 5 to $7 \mathrm{ps,} \mathrm{showing}$ multiple time constants. There is no consensus on the assignment of these processes, with a dispute between models claiming that thymine is trapped either in the first $\left(\mathrm{S}_{1}\right)$ or in the second $\left(\mathrm{S}_{2}\right)$ excited states. In the present study, a nonadiabatic dynamics simulation of thymine is performed on the basis of ADC(2) surfaces, to understand the role of dynamic electron correlation on the deactivation pathways. The results show that trapping in $S_{2}$ is strongly reduced in comparison to previous simulations considering only non-dynamic electron correlation on CASSCF surfaces. The reason for the difference is traced back to the energetic cost for formation of a $\mathrm{CO} \pi$ bond in $\mathrm{S}_{2}$.
\end{abstract}

Keywords: computational theoretical chemistry; photochemistry; nonadiabatic dynamics; ultrafast processes; surface hopping; nucleobases; thymine

\section{Introduction}

After UV excitation, gas phase thymine returns to the ground state within 5 to $7 \mathrm{ps}$ [1]. In the 14 years since ultrafast time-resolved spectroscopy of this molecule was reported for the first time [2], this seems to be the only consensus on the interpretation of its photophysics. The elusive nature of thymine's photophysics stems from the difficulty of assigning multiple time constants underlying its time-resolved photoelectron spectrum [1-10]. In fact, a literature survey (see Table 1) reveals that there is no full agreement on even how many time constants are implicit in those spectra [1,3,7]. Most of results tend to converge to a three time-constants scheme, with a short sub-picosecond time constant of about 100-200 fs, a picosecond time constant of about 6 ps, and a nanosecond time constant reaching nearly $300 \mathrm{~ns}$.

Taking the picosecond time constant as an indication of internal conversion to the ground state-which is the most common interpretation-leaves thymine with the longest excited state lifetime among the isolated nucleobases $[7,11]$. This fact is in itself puzzling, as thymine's potential energy surfaces obtained from high-level computational simulations are very similar to those of other short-lived pyrimidines (uracil, for instance), to justify the time constant differences [12]. 
Table 1. Excited-state time constants of thymine in the gas phase according to the experiments under various pump and probe conditions.

\begin{tabular}{ccccccc}
\hline Pump $(\mathbf{n m})$ & Probe $(\mathbf{n m})$ & $\left.\boldsymbol{\tau}_{\mathbf{1}} \mathbf{( f s}\right)$ & $\boldsymbol{\tau}_{\mathbf{2}} \mathbf{( p s )}$ & $\boldsymbol{\tau}_{\mathbf{3}}(\mathbf{p s})$ & $\boldsymbol{\tau}_{\mathbf{4}} \mathbf{( n s )}$ & Reference \\
\hline 250 & 200 & $<50$ & 0.49 & 6.4 & & {$[3]$} \\
260 & 295 & 175 & & 6.13 & $>1$ & {$[1]$} \\
266 & $2.19($ X-ray) & $200-300$ & & & & {$[4]$} \\
266 & $400 / 800$ & $<100$ & & 7 & long & {$[5]$} \\
266 & 800 & 200 & & 7 & & {$[6]$} \\
267 & $2 \times 400$ & 105 & & 5.12 & & {$[7]$} \\
267 & 800 & 100 & & 7 & $>1$ & {$[8]$} \\
267 & 800 & & & 6.4 & $>100$ & {$[2]$} \\
270 & 193 & & & & 293 & {$[9]$} \\
272 & 800 & 130 & & 6.5 & & {$[10]$} \\
\hline
\end{tabular}

Computational simulations have revealed that thymine internal conversion after UV excitation should involve two singlet excited adiabatic states, $S_{1}$ and $S_{2}[12,13]$. These states may have $n \pi^{*}$ or diverse $\pi \pi^{*}$ characters along the reaction paths. There is an extended accessible crossing seam region between $S_{2}$ and $S_{1}\left(\pi \pi^{*} / n \pi^{*}\right)$ [14], as well as between $S_{1}$ and the ground state $\left(\pi \pi^{*} / S_{0}\right.$ and $\left.n \pi^{*} / S_{0}\right)$ [15]. A long-lived triplet $\pi \pi^{*}$ state plays a role over longer scales $[1,9,16,17]$ not explored here.

In earlier works, thymine's shortest time constant has been assigned to direct internal conversion to ground state along a $\pi \pi^{*}$ pathway. Such a model—we will refer to it as the "fast $\pi \pi^{*}$ model" — was proposed on the basis of either analyses of ab initio potential energy surfaces $[18,19]$ or surface hopping dynamics on semi-empirical surfaces [20]. Nevertheless, the agreement between these works is restricted to this sub-picosecond step: while ref. [18] proposes that the picosecond step would occur due to a delayed $\pi \pi^{*}$ deactivation, ref. [19] attributes this longer step to a sequential $\pi \pi^{*} \rightarrow \mathrm{n} \pi^{*} \rightarrow \mathrm{S}_{0}$ conversion. Ref. [20], on its turn, also predicts a sequential $\pi \pi^{*} \rightarrow \mathrm{n} \pi^{*} \rightarrow \mathrm{S}_{0}$ conversion process, but occurring in the sub-picosecond scale.

A different photophysical model was proposed in ref. [13] and later corroborated by ref. [15], both on the basis of analysis of ab initio potential energy surfaces. This model-the " $\mathrm{S}_{1}$ trapping model" - assigns the short time constant to a fast $S_{2}\left(\pi \pi^{*}\right) \rightarrow S_{1}\left(n \pi^{*}\right)$ transition, while the picosecond time constant is assigned to a $S_{1}\left(n \pi^{*}\right) \rightarrow S_{0}$ transition. Thus, according to this interpretation, the elongated picosecond time constant of thymine would be caused by a trapping in the $\mathrm{S}_{1}$ state.

The $S_{1}$ trapping model has been popular among experimentalists, as it apparently correlates well with the electron binding energy $\left(E_{b}\right)$ observed in time-resolved experiments $[1,6,21]$. Their argument goes as follows: the first ionization potential (IP) of thymine is a $\pi$ hole, while the second is an $n$ hole. Thus, spectral signals at low $E_{b}$ near the first IP should be caused by probing the $\pi \pi^{*}$ state, while spectral signals at large $E_{b}$ near the second IP should be caused by probing the $\mathrm{n} \pi^{*}$ state. Because the signal in the picosecond scale comes from large $E_{b}$, this would be an evidence that thymine is in the $\mathrm{n} \pi^{*}$ state during the picosecond regime. The problem with this argument is that it assumes that electrons are usually ejected with the maximum electron kinetic energy (or minimum $E_{b}$, near the IP). This is correct only for ionization of stationary states. When probing wave packets, a much wider range of electron kinetic energies should be expected [22]. Thus, while it is true that spectral signal near the first IP should be essentially due to $\pi \pi^{*}$ probing, the signal near the second IP contains not only information from $n \pi^{*}$ probing, but also information from $\pi \pi^{*}$ probing of electrons being ejected with low kinetic energy.

Although this analysis of the electron kinetic energy does not disprove the $S_{1}$ trapping model (which is good for us, as will be advocating for it later), it at least reduces its strength. If that were not enough, there is still a third model for thymine deactivation in direct competition with it, the "S $\mathrm{S}_{2}$ trapping model".

$\mathrm{S}_{2}$ trapping was first proposed on the basis of multiple spawning dynamics on complete active space self-consistent field (CASSCF) surfaces [23]. These simulations, limited to a short sub-picosecond time scale, showed that after excitation into $S_{2}\left(\pi \pi^{*}\right)$ state, conversion to $S_{1}\left(n \pi^{*}\right)$ was unexpectedly slow. This led to the hypothesis that the picosecond time constant was due to thymine's trapping in $S_{2}$, 
while the short sub-picosecond time constant was caused by relaxation of the $\pi \pi^{*}$ state between the Franck-Condon region and the $S_{2}$ minimum.

The $S_{2}$ trapping model got some additional support from surface hopping dynamics still on CASSCF surfaces $[14,24]$. These simulations were performed on longer time scales than in the original multiple spawning simulations and confirmed that slow $S_{2} \rightarrow S_{1}$ transfer. However, the surface hopping results also added a new layer of complexity, as they showed that the $S_{2}$ trapping could only explain a delay of about 2 ps in the lifetime; therefore, to reach a 6 ps time constant, thymine should also be trapped in $S_{1}$ after the $S_{2} \rightarrow S_{1}$ transition. A final bit of complexity was later added to the model by wave packet dynamics [25]. It showed that even the common hypothesis that only the $\pi \pi^{*}$ state is excited needs to be relaxed, as vibronic couplings could lead to a substantial $n \pi^{*}$ population within the first $50 \mathrm{fs}$ of dynamics, with the remaining $\pi \pi^{*}$ population trapped in a flat $S_{2}$. Thus, together, these results from surface hopping and wave packet dynamics seemed to point out to a new " $\mathrm{S}_{2}$ and $\mathrm{S}_{1}$ trapping model".

A couple of years ago, however, the $S_{2}$ trapping hypothesis was challenged by time-resolved Auger spectroscopy [4], which combined with spectrum simulations at CIS level made a good case towards a population transfer to the $n \pi^{*}$ state within 200-300 fs. Once more, the $S_{1}$ trapping model would be invoked to explain the picosecond time constant.

Giving this cloudy state of affairs, we decided to revisit thymine dynamics. Although multiple spawning and surface hopping dynamics have provided some compelling arguments for the $\mathrm{S}_{2}$ trapping, these simulations have a common major weak point: they were based on CASSCF surfaces. CASSCF does an excellent job recovering non-dynamic electron correlation near intersections between the ground and the first excited states, but it neglects most dynamic electron correlation, which is present through the whole reaction path. This poses a serious problem: the key step to determine the occurrence (or not) of the $S_{2}$ trapping is the $S_{2}$ dynamics up to the $S_{2} / S_{1}$ crossing. In this region of the potential energy surface, we do not expect any relevant impact of non-dynamic electron correlation, but we are sure that dynamic electron correlation plays a role; for instance, correcting the strong overestimation of the $\pi \pi^{*}$ energy typical of CASSCF predictions $[15,19]$. Therefore, we have approached the problem through surface hopping simulations based on algebraic diagrammatic construction to second order (ADC(2)) method, which, quite opposite to CASSCF, effectively recovers dynamic correlation but neglects non-dynamic correlation. We can already anticipate that this methodological change had a major impact on the results: the $S_{2}$ trapping is strongly reduced.

\section{Results}

\subsection{Topography of Excited States}

Thymine's vertical excitation at ADC(2)/(aug-)cc-pVDZ level is characterized by a dark $\mathrm{S}_{1}$ state at $4.56 \mathrm{eV}$ with $n \pi^{*}$ character and a bright $\mathrm{S}_{2}$ excitation at $5.06 \mathrm{eV}$ with a $\pi \pi^{*}$ character (Table 2). Electronic density differences for these two states in comparison to the ground state density are shown in Figure 1.

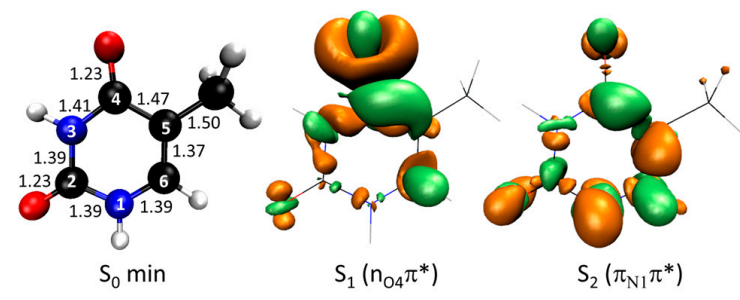

Figure 1. (Left) Geometry of ground state thymine with atom numbering and main bond lengths in $\AA$; (Center) Difference between the electronic densities of the $S_{1}$ state $\left(n \pi^{*}\right)$ and of the ground state; (Right) Difference between the electronic densities of the $S_{2}$ state $\left(\pi \pi^{*}\right)$ and of the ground state. In this figure and throughout the paper, orange surfaces in the density difference indicate electron deficient regions, while green surfaces indicate electron rich regions. 
Table 2. Ground and excited singlet state energies of the minima and intersection points of thymine in the gas phase obtained with ADC(2), CASSCF, and MS-CASPT2. All energies are relative to the ground state minimum.

\begin{tabular}{ccccc}
\hline \multirow{2}{*}{ Geometry } & State & \multicolumn{3}{c}{ Energy (eV) } \\
\cline { 3 - 5 } & & ADC(2) & CASSCF $^{\mathbf{a}}$ & MS-CASPT2 $^{\mathbf{b}}$ \\
\hline \multirow{3}{*}{$\mathrm{S}_{0} \min$} & $\mathrm{S}_{0}(\mathrm{cs})$ & 0.00 & 0.00 & 0.00 \\
& $\mathrm{~S}_{1}\left(\mathrm{n}_{\mathrm{O} 4} \pi^{*}\right)$ & 4.56 & 5.19 & 5.09 \\
& $\mathrm{~S}_{2}\left(\pi_{\mathrm{N} 1} \pi^{*}\right)$ & 5.06 & 6.87 & 5.09 \\
\hline \multirow{2}{*}{$\mathrm{S}_{1} \min$} & $\mathrm{S}_{0}(\mathrm{cs})$ & 1.33 & 1.39 & 1.02 \\
& $\mathrm{~S}_{1}\left(\mathrm{n}_{\mathrm{O} 4} \pi^{*}\right)$ & 3.33 & 4.02 & 4.37 \\
\hline \multirow{2}{*}{$\mathrm{S}_{2} \min$} & $\mathrm{S}_{0}(\mathrm{cs})$ & 2.14 & 1.71 & 1.28 \\
& $\mathrm{~S}_{1}\left(\mathrm{n}_{\mathrm{O} 4} \pi^{*}\right)$ & 3.50 & 4.18 & 4.51 \\
& $\mathrm{~S}_{2}\left(\pi_{\mathrm{O} 4} \pi^{*}\right)$ & 4.18 & 5.64 & 4.77 \\
\hline \multirow{2}{*}{$\mathrm{X}_{10}\left(\mathrm{n} \pi^{*} / \mathrm{S}_{0}\right)$} & $\mathrm{S}_{0}(\mathrm{cs})$ & 3.90 & 5.02 & 5.02 \\
& $\mathrm{~S}_{1}\left(\mathrm{n}_{\mathrm{O} 4} \pi^{*}\right)$ & 3.90 & 5.13 & 5.60 \\
\hline \multirow{2}{*}{$\mathrm{X}_{10}\left(\pi \pi^{*} / \mathrm{S}_{0}\right)$} & $\mathrm{S}_{0}(\mathrm{cs})$ & 3.82 & 4.49 & 4.19 \\
& $\mathrm{~S}_{1}\left(\pi_{56} \pi^{*}\right)$ & 3.82 & 5.54 & 4.41 \\
\hline \multirow{2}{*}{$\mathrm{X}_{21}\left({ }^{3,6} \mathrm{~B}\right)$} & $\mathrm{S}_{0}(\mathrm{cs})$ & 3.37 & 2.68 & 2.23 \\
& $\mathrm{~S}_{1}\left(\mathrm{n}_{\mathrm{O} 4} \pi^{*}\right)$ & 4.21 & 5.61 & 4.79 \\
& $\mathrm{~S}_{2}\left(\pi_{56} \pi^{*}\right)$ & 4.22 & 6.00 & 5.63 \\
\hline
\end{tabular}

a CASSCF(12,9)/6-311G* and ${ }^{\mathrm{b}}$ MS-CASPT2(12,9)/6-311G* on CASSCF(8,6)/6-31G* geometries; data from ref. [19].

The main topographic points in these two excited states are the minima on $S_{2}$ and $S_{1}$, the intersection point between $S_{2}$ and $S_{1}$, and the two intersection points between $S_{1}$ and $S_{0}$. They are characterized in Figure 2. Like in the Franck-Condon (FC) region, the $S_{2}$ state around the $S_{2}$ minimum has a $\pi \pi^{*}$ character. Nevertheless, while in the FC region the electron is promoted from a $\pi$ bond involving $\mathrm{N} 1, \mathrm{C} 5$, and $\mathrm{C} 6$, in the $\mathrm{S}_{2}$ minimum the electron is promoted from the $\mathrm{C} 4 \mathrm{O} \pi$ bond (compare the electronic density differences in Figures 1 and 2). As a consequence of losing the $\mathrm{C} 4 \mathrm{O} \pi$ bond in the $\mathrm{S}_{2}$ minimum, there is a strong stretching of the $\mathrm{C} 4 \mathrm{O}$ distance from $1.23 \AA$ in the FC region to $1.48 \AA$ in the $S_{2}$ minimum. We will later discuss how this feature has a major impact on the $S_{2} \rightarrow S_{1}$ dynamics. Another feature of this minimum is a shrinking of the $\mathrm{C} 4 \mathrm{C} 5$ and $\mathrm{C} 5 \mathrm{C} 6$ bonds, indicating the formation of $\pi$ bonds in that region.
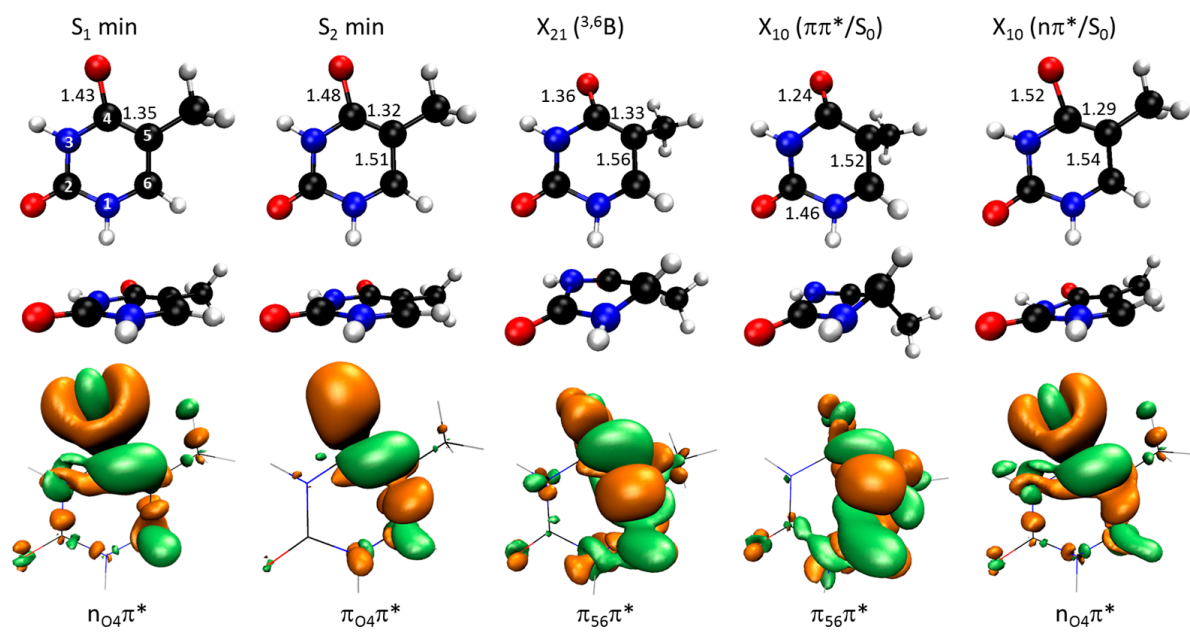

Figure 2. Geometries of the $S_{1}$ and $S_{2}$ minima, and of the $X_{21}, X_{10}\left(\pi \pi^{*} / S_{0}\right)$ and $X_{10}\left(n \pi^{*}\right)$ intersection points. The bond distances with the largest variation in comparison to the ground state geometry are given in $\AA$. The electronic density difference between the relevant state in each case and the ground state are shown at the bottom. 
The $S_{1}$ state in the $S_{1}$ minimum still has the same $n \pi^{*}$ character as in the FC region (electron excitation from $\mathrm{C} 4 \mathrm{O}$ ). Compared to the ground state geometry, the main geometric consequence of the relaxation into this minimum is the stretching of the $\mathrm{C} 4 \mathrm{O}$ bond and the shrinking of the $\mathrm{C} 4 \mathrm{C} 5$.

The crossing between $S_{2}$ and $S_{1}$ is reached by an out-of-plane deformation of the ring (Figure 2). At the minimum energy crossing point, the ring assumes a boat conformation with N3 and C6 above the plane $\left({ }^{3,6} \mathrm{~B}\right)$. Along the $S_{2}$ state, this crossing still occurs on a $\pi \pi^{*}$ state, but there is a significant density change in comparison to that of the $S_{2}$ minimum. While in the $S_{2}$ minimum the $C 4 \mathrm{O} \pi$ bond is lost, in the $\mathrm{X}_{21}$ crossing this bond it is recovered. This is clear from the shrinking of the C4O distance from 1.48 to $1.36 \AA$ between these two geometries. In fact, it is exactly this bond formation responsible for the energy stabilization, which ultimately leads to the intersection.

The character change of the $\pi \pi^{*}$ state between the FC region and the $S_{2}$ minimum was first pointed out in ref. [21], while the character change between the $S_{2}$ minimum and the $X_{21}$ intersection was first noticed in ref. [14]. Both works, however, were limited to an analysis of the main molecular orbitals involved in the transitions. The density difference analysis goes a step further, revealing more precisely where the excitations originated.

There are two main minimum energy crossings between $S_{1}$ and $S_{0}$. The first one connects the $\pi \pi^{*}$ state to the ground state $\left(\mathrm{X}_{10} \pi_{56} \pi^{*} / \mathrm{S}_{0}\right.$ in Figure 2$)$. It occurs along the same type of geometrical distortion that gives rise to $\mathrm{X}_{21}$. The $\mathrm{X}_{10} \pi \pi^{*} / \mathrm{S}_{0}$ crossing also features a ${ }^{3,6} \mathrm{~B}$ boat conformation, but while the puckering degree is $Q=0.48 \AA$ for $X_{21}$, it increases further to $Q=0.54 \AA$ for $X_{10}(Q$ is the Cremer-Pople parameter measuring the degree of puckering in a 6-membered ring [26]). At the crossing, the $\mathrm{C} 4 \mathrm{O} \pi$ bond is fully formed and the $\mathrm{C} 4 \mathrm{O}$ distance is $1.24 \AA$, essentially the same as in the ground state, $1.23 \AA$.

The second $X_{10}$ crossing connects the $n \pi^{*}$ state to the ground state $\left(X_{10} n_{O 4} \pi^{*} / S_{0}\right.$ in Figure 2). It occurs as a further semi-planar distortion of the $\mathrm{S}_{1}$ minimum, with the $\mathrm{C} 4 \mathrm{O}$ bond stretched to $1.52 \AA$ and the C4C5 bond shrank to $1.29 \AA$.

This general topography of the lowest singlet excited states is illustrated in Figure 3. The top graph is the potential energy profile of the $S_{0}, S_{1}$, and $S_{2}$ states obtained by linear interpolation of internal coordinates (LIIC) between the two $X_{10}$ intersection points. The bottom graph shows $S_{1}$ and $S_{2}$ along the interpolation between the $S_{2}$ minimum and the $X_{21}$ intersection.

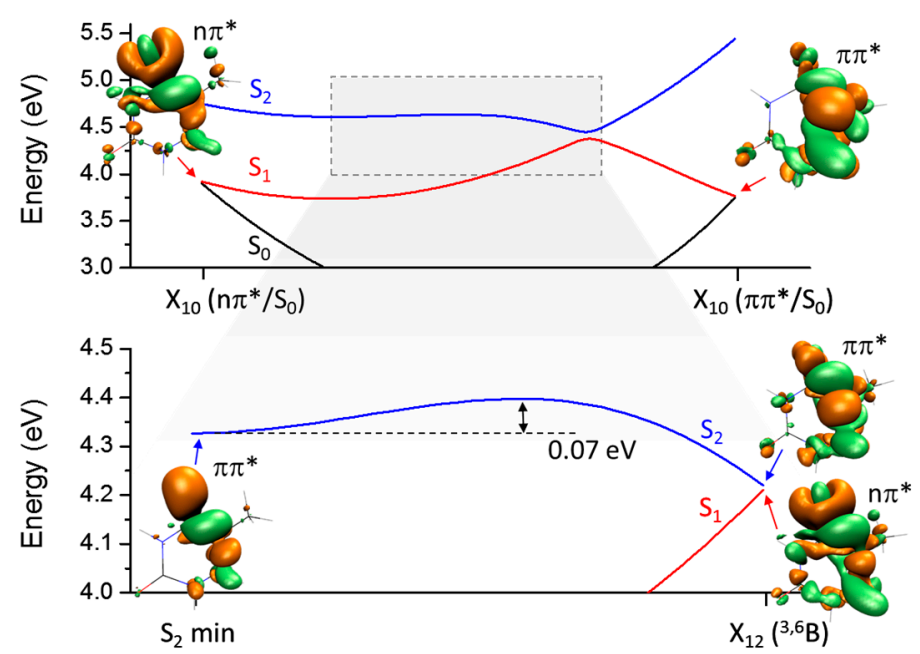

Figure 3. (Top) LIIC profile between the two $X_{10}$ intersection points; (Bottom) LIIC profile between the $S_{2}$ minimum and the $X_{21}$ intersection point. Electronic density differences at key points are shown as well.

As already mentioned, starting from the $S_{2}$ minimum, $X_{12}$ is reached by an out-of-plane distortion that recovers the $\mathrm{C} 4 \mathrm{O}$ bond. With $\mathrm{ADC}(2)$, the cost for this bond formation is minimum, only $0.07 \mathrm{eV}$. 
For comparison, at CASSCF, the same interpolated barrier is $0.35 \mathrm{eV}$ [14]. Note that these are linearly interpolated values, which overestimate the true barriers. Full optimization of transition states resulted in barriers of $0.25 \mathrm{eV}$ with CASSCF [19] and between 0.01 and $0.05 \mathrm{eV}$ with multi-state complete active space perturbation theory to second order (MS-CASPT2) [12,19].

Although the qualitative description of the excited state topography of thymine obtained with $\mathrm{ADC}(2)$ is in agreement with previous description using other computational methods $[1,15,19]$, it is clear from Table 2 that this agreement is merely qualitative. The quantitative description of the minima and intersection energies bears important differences between the methods. Unfortunately, at this point we cannot take for granted even that CASPT2 result would be the most accurate, as the usual protocol of computing CASPT2 energies on CASSCF optimized geometries may result in poor excitation energies, especially near the crossing seam (see, for instance, in Table 2, the large energy splits when MS-CASPT2 is used on CASSCF optimized intersection geometries). With this methodological warning in mind, we will present the dynamics results in the next section and later discuss possible sources of inaccuracy on the ADC(2) surfaces.

\subsection{Dynamics}

Initial conditions for dynamics were obtained by first simulating the absorption spectrum of thymine in the gas phase. This spectrum is shown in Figure 4 compared to the experimental result in water from ref. [27]. The ADC(2)/(aug)-cc-pVDZ absorption band is peaked at $4.89 \mathrm{eV}$. The experimental gas phase result obtained by electron impact is $4.95 \pm 0.08 \mathrm{eV}$ [28]. The absorption intensity and band shape are also in very good agreement with the experimental results in water [27].

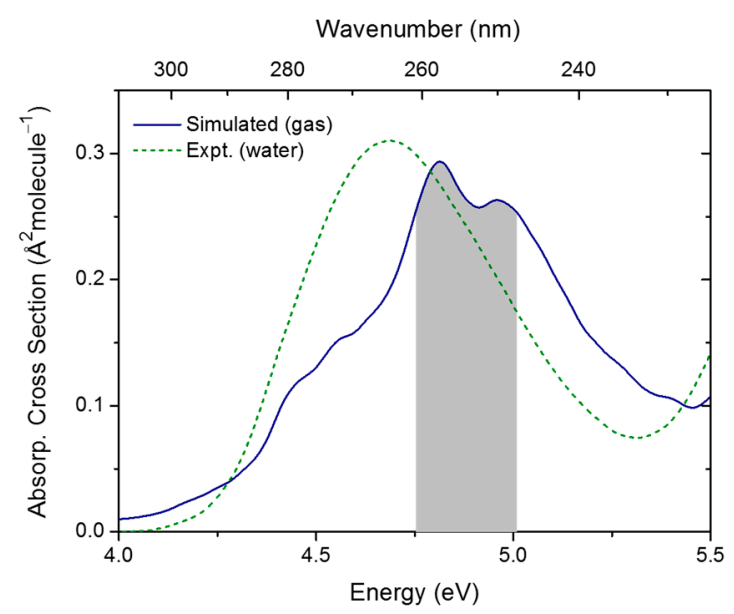

Figure 4. Simulated spectrum of thymine in the gas phase. The shaded area indicates where initial conditions for dynamics where selected from. The dashed line is the experimental spectrum of thymine in water from ref. [27].

ADC(2)/(aug-)cc-pVDZ surface hopping dynamics of thymine in the gas phase shows a fast relaxation process, with $S_{2}$ converting to $S_{1}$, and then $S_{1}$ converting to $S_{0}$ (Figure 5). The fitting of the state occupation (fraction of trajectories in each state) as a function of time shows an $S_{2} \rightarrow S_{1}$ exponential decay of $84 \%$ of the population within 253 fs (Table 3). The fitting of the $S_{1}$ occupation (see Supplementary Materials) reveals that $70 \%$ of the population returns to the ground state with a $391 \mathrm{fs}$ time constant. $30 \%$ of the total population deactivates with a time constant above 1 ps. Note that considering a confidence level of $90 \%$, our 115 trajectories only allow these fractions to be determined within a maximum statistical uncertainty of $\pm 8 \%$. 
Table 3. Time constants for different processes and corresponding fractions of population being affected by them. For the $S_{2} \rightarrow S_{1}$ and $S_{1} \rightarrow S_{0}$ processes, parameters were obtained by fitting the state occupations in Figure 5 with the kinetic model discussed in the Supplementary Material. For FC $\rightarrow \mathrm{S}_{2}$ min, the information was extracted from Figure 6.

\begin{tabular}{ccc}
\hline Process & $f_{\boldsymbol{\tau}}$ & $\boldsymbol{\tau}(\mathbf{f s})$ \\
\hline $\mathrm{FC} \rightarrow \mathrm{S}_{2} \min$ & 1.00 & $\sim 100$ \\
$\mathrm{~S}_{2} \rightarrow \mathrm{S}_{1}$ & 0.84 & 253 \\
$\mathrm{~S}_{1} \rightarrow \mathrm{S}_{0}$ & 0.70 & 391 \\
\hline
\end{tabular}

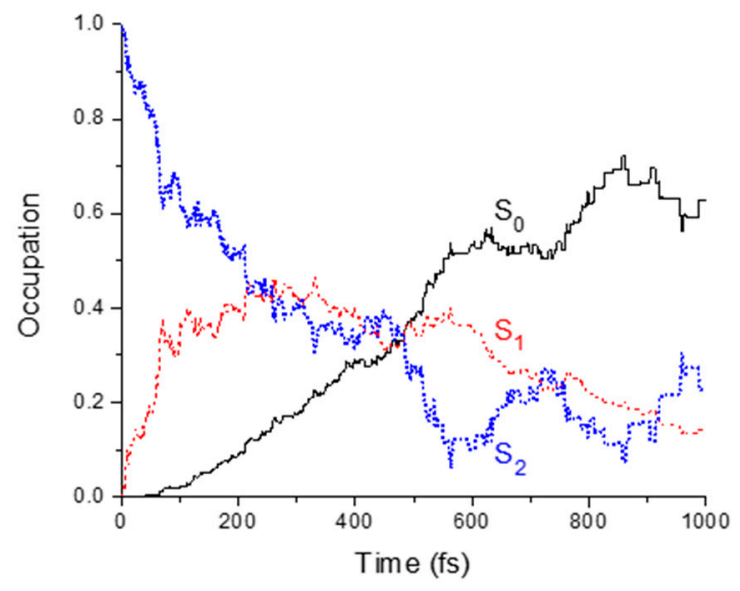

Figure 5. State occupations during dynamics.
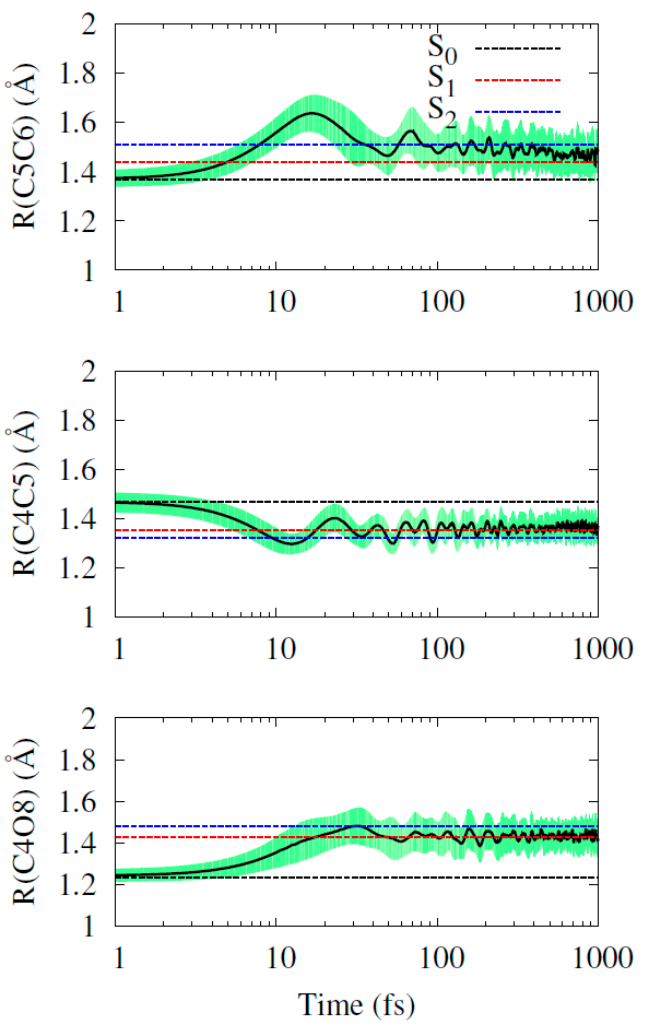

Figure 6. Time evolution of the C5C6 (Top), C4C5 (Middle), and C4O (Bottom) bond distances averaged over all trajectories. The shaded areas show plus-minus one standard deviation around the mean value. Horizontal lines indicate the optimal values of the $S_{0}, S_{1}$ and $S_{2}$ minima. 
As we discussed in the previous section, the $\mathrm{C} 5 \mathrm{C} 6, \mathrm{C} 4 \mathrm{C} 5$, and $\mathrm{C} 4 \mathrm{O}$ bond distances are markedly distinct in the three state minima. Therefore, their evolution during the dynamics is useful to gather further information on the state population. The time evolution of these bond distances averaged over all trajectories are shown in Figure 6 . All three start near the optimal $S_{0}$ minimum value. The $S_{2}$ minimum is quickly reached, after $100 \mathrm{fs}$. This can be clearly seen only in the C5C6 bond, which bears the largest difference between $S_{1}$ and $S_{2}$ minima. In the other two cases, the large number of trajectories quickly decaying to $S_{1}$ (together with the large standard deviation) tends to hide this feature. By the end of the simulations, the three bond distances oscillate near the $S_{1}$ minimum. (As we discuss in the Theoretical and Computational Details, we do not simulate the ground state dynamics. For this reason, in the long term, we do not see the ground state bond distances being recovered.)

The $S_{2} \rightarrow S_{1}$ conversion occurs in a wide variety of ring puckering conformations, including distortions far away from the minimum intersection point. This is illustrated in Figure 7, which shows the distribution of Cremer-Pople parameters $\theta$ and $\phi$ at the $S_{2} / S_{1}$ hop point. (These two parameters characterize the type of puckering in a 6-membered ring.) Larger ring distortions (large $Q$ ) tend to occur near the ${ }^{3,6} \mathrm{~B}$ region $\left(\theta=90^{\circ}, \phi=120^{\circ}\right)$. There is no correlation between the type of ring puckering and the hop time.
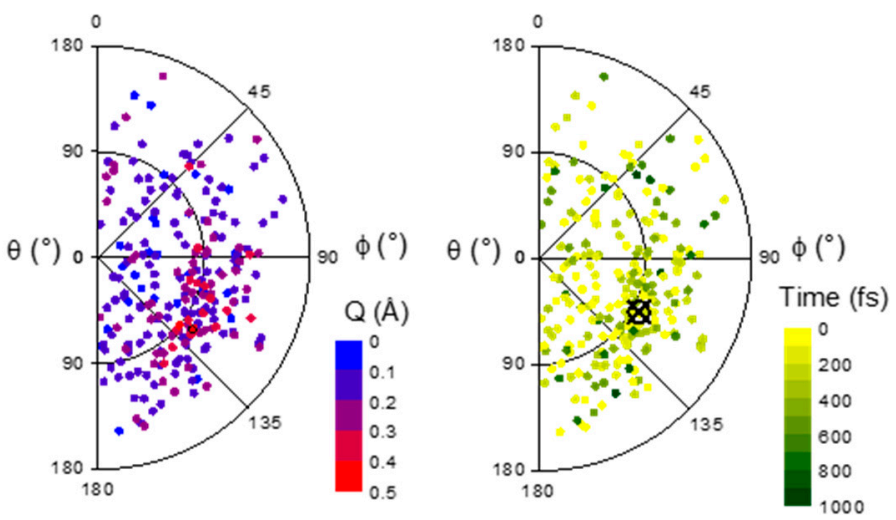

Figure 7. Polar plot showing the distribution of Cremer-Pople parameters $\theta$ and $\phi$ at the $S_{2} / S_{1}$ hop geometry. On the left, the colors additionally indicate the value of the third parameter $Q$. On the right, the color code indicates the hop time. Both maps were symmetry-projected to show only the $\phi<180^{\circ}$ region. The crossed circle indicates the minimum energy crossing point.

The $\mathrm{S}_{1} \rightarrow \mathrm{S}_{0}$ conversion occurs at both branches of intersection, the $\mathrm{n} \pi^{*} / \mathrm{S}_{0}$ and the $\pi \pi^{*} / \mathrm{S}_{0}$. From the $84 \%$ of the population converting to $S_{1}, 61 \%$ deactivates in the $n \pi^{*} / S_{0}$ crossing and $9 \%$ in the $\pi \pi^{*} / S_{0}$. Finally, $14 \%$ of the population does not decay in the sub-picosecond process and remains in $S_{1}$.

\section{Discussion}

The results of the ADC(2) surface hopping dynamics of thymine in the gas phase are schematically summarized in Figure 8. After photoexcitation into the $\pi_{\mathrm{N} 1} \pi^{*}$ state (a), thymine relaxes within $100 \mathrm{fs}$ to the minimum of the $S_{2}$ surface holding a $\pi_{\mathrm{O} 4} \pi^{*}$ character $(\mathrm{b})$. A minor fraction of the population is trapped in $S_{2}$ (c), while the remaining flows to $S_{1}$ in about $250 \mathrm{fs}(\mathrm{d})$. This conversion to $S_{1}$ splits the population once more: a minor part follows the $S_{1}$ state along the $\pi_{56} \pi^{*}$ branch and immediately converts to the ground state (e); the major part, however, flows to the $S_{1} n_{\mathrm{O} 4} \pi^{*}$ minimum (f). After about $400 \mathrm{fs}$, most of the population converts to the ground state in the $\mathrm{n}_{\mathrm{O} 4} \pi^{*} / \mathrm{S}_{0}$ crossing $(\mathrm{g})$, while a minor fraction remains trapped in the $S_{1}$ state $(h)$. 


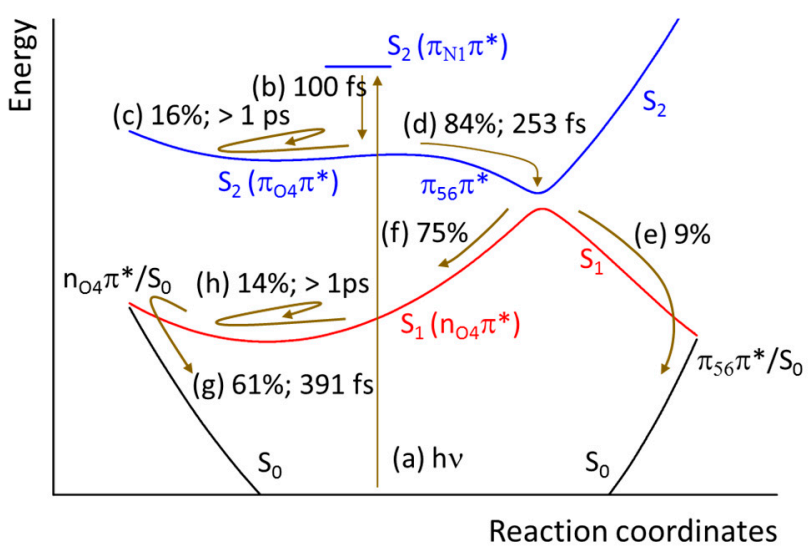

Figure 8. Schematic view of thymine dynamics as predicted by ADC(2) surface hopping. See text for description.

These results imply that, upon inclusion of dynamic electron correlation in the dynamics, the $\mathrm{S}_{2}$ trapping is drastically reduced and may affect only $16 \%$ of the population. In CASSCF dynamics, it affects about $80 \%$ of the population [14]. This difference is a strong indication that dynamics based on CASSCF $[14,23]$ may have overestimated the role of the $S_{2}$ trapping. The reason for this overestimation is clear: in CASSCF the formation of $\mathrm{C} 4 \mathrm{O} \pi$ bond (which allows to reach the $\mathrm{S}_{2} / \mathrm{S}_{1}$ intersection) has an energetic cost, in the form of a barrier $\left(0.25 \mathrm{eV}\right.$ [19]) separating the $S_{2}$ minimum and the intersection. This barrier practically disappears when dynamic electron correlation is included, either in ADC(2) or in CASPT2.

$\mathrm{ADC}(2)$ is a single reference method, whose current implementation is based on linear response theory. Naturally, we cannot expect that it will provide definitive answers on thymine time constants. Moreover, we should consider that we cannot accurately compute the time constant for deactivation to $S_{0}$ due to the lack of $S_{1} / S_{0}$ nonadiabatic couplings. As explained later in the section Theoretical and Experimental Details, we deal with this problem using an energy threshold as hop criterion. For this reason, both the $\mathrm{S}_{1} \rightarrow \mathrm{S}_{0}$ time constant and fraction of population bear large uncertainties. For instance, if we double the energy gap threshold from 0.15 to $0.30 \mathrm{eV}$, the $\mathrm{S}_{1} \rightarrow \mathrm{S}_{0}$ time constant is reduced from 391 to $291 \mathrm{fs}$.

In particular, the efficient $S_{1} / S_{0}$ conversion of $70 \%$ of the population in the sub-picosecond scale is especially challenging to rationalize in view of the experimental signal in the few picoseconds range (Table 1). Even if the third of the population which is left in the excited states decayed with a time constant spanning a few picoseconds, this fraction may be too small to account for the strong ion signal originating from this spectral region. Nevertheless, without a full spectral simulation including the probe process, we also cannot discard the possibility that this third of the population is in fact ultimately responsible for the signal. Unfortunately, the experimental references do not disclose the fitting amplitudes in addition to the time constants. They would be invaluable to check this point.

If the fraction of the population decaying in the picosecond scale is significantly larger than $30 \%$, this will indicate that the $n \pi^{*} / \mathrm{S}_{0}$ intersection predicted by ADC(2) is too low in energy, which could be result of the wrong topography of the $S_{1} / S_{0}$ crossing seam at this level [29]. However, even if we conclude that $\mathrm{ADC}(2)$ dynamics is artificially fast, it seems improbable that its prediction of sub-picosecond $S_{1} / S_{0}$ conversion is completely wrong. The occurrence of this fast process in thymine should be seriously considered, as it has recurrently shown up in the simulations: it is relevant in ADC(2) dynamics, dominant in semi-empirical OM2/MRCI dynamics [20], and even in CASSCF dynamics it affects about $20 \%$ of the population [14]. In practical terms, this means that the current trend of fitting time-resolved spectra of thymine with three exponential decays with fs, ps, and ns time constants may be too strict. We may even recall alternative fittings, like that in ref. [3], which split the sub-picosecond time constant in two, $<50 \mathrm{fs}$ and $490 \mathrm{fs}$. 
The photodynamics of thymine has daring experimentalists and theoreticians. Although we are still not in a position to deliver a final assignment of its many spectral features, it is becoming obvious that assigning its time constants to single processes may be the wrong strategy. The ensemble of results points to a situation where several processes contribute to the dynamics in the same time scale. In particular, it is astonishing that in the sub-picosecond time scale alone, the time-resolved spectra may be influenced by laser field, variation of the IP along $S_{2}$ relaxation through three different $\pi \pi^{*}$ characters, $S_{2} / S_{1}$ conversion, and $S_{1} / S_{0}$ conversion in two different branches of the crossing seem.

To learn how to resolve each of them is the next challenge.

\section{Theoretical and Computational Details}

\subsection{Potential Energy, Spectrum, and Dynamics Simulations}

The geometries of the ground and the first two singlet excited states of thymine were optimized with algebraic diagrammatic construction to second order (ADC(2)) level [30,31] (for the ground state, on MP2 level). The Dunning's aug-cc-pVDZ basis set was used for all elements except for hydrogen, where cc-pVDZ was employed [32]. This mixed basis set is denoted (aug-)cc-pVDZ in the text. Calculations were done with frozen core and applying the resolution-of-identity (RI) approximation for the computation of two-electron integrals. In addition to state minima, we also optimized two intersection minima between $S_{0}$ and $S_{1}$ states (denoted $X_{10}$ ), and an intersection minimum between $S_{2}$ and $S_{1}$ (denoted as $X_{21}$ ). Reaction paths were computed applying linear interpolation in natural internal coordinates (LIIC) [33].

We simulated the photoabsorption spectrum of thymine applying the nuclear ensemble approach [34]. A set of 500 molecular geometries and momenta was created using harmonic-oscillator Wigner distribution based on normal modes in the ground state. Vertical excitation energies and oscillator strengths for transitions to the first ten singlet states were computed using $\mathrm{ADC}(2) /($ aug-)cc-pVDZ for each geometry in the ensemble.

We performed nonadiabatic excited-state dynamics simulations using surface hopping on $\mathrm{ADC}(2) /$ (aug-)cc-pVDZ potential energy surfaces. The initial conditions (geometries and momenta) for dynamics simulations were selected starting from the bright $S_{2}$ state. They were filtered from the initial ensemble of 500 initial conditions, from within the $4.88 \pm 0.13 \mathrm{eV}$ energy window, which includes the maximum of the first band in the spectrum. This procedure produced 115 initial conditions, which were propagated for a maximum 1 ps.

Nonadiabatic events between $S_{2}$ and $S_{1}$ were taken into account by the fewest switches algorithm [35] corrected for decoherence effects $(\alpha=0.1$ Hartree) [36]. Because of the limitation of $\mathrm{ADC}(2)$ to deal with multi-reference ground states [29], trajectories were stopped whenever their $\mathrm{S}_{1} / \mathrm{S}_{0}$ energy gap dropped below $0.15 \mathrm{eV}$. The corresponding time step was taken as an estimate of the $S_{1} / S_{0}$ crossing time. Newton's equations of motion were integrated using the velocity Verlet algorithm [37] with the time step of $0.5 \mathrm{fs}$. Integration of the semi-classical Schrödinger equation was done employing the 5th order Butcher's algorithm [38] with time step of $0.025 \mathrm{fs}$, using interpolated electronic properties between the classical steps. Computation of nonadiabatic couplings between excited states is described in the next section.

To analyze the distortions of thymine's ring during dynamics, we computed the Cremer-Pople parameters [26] and classified them into conformations according to Boeyens' scheme [39].

All ADC(2) computations were done with TURBOMOLE [40]. Spectrum and dynamics were computed with the NEWTON-X/TURBOMOLE interface [41,42]. Intersection point optimizations were done with an in-house modified version of CIOpt program [43]. Cremer-Pople parameters were obtained using the PLATON program [44]. 


\subsection{OD Method for Coupling Calculations}

Nonadiabatic couplings $\sigma_{m n}$ between electronic states $m$ and $n$ can be dynamically estimated on the basis of the time derivative of the corresponding wave functions during the trajectory:

$$
\sigma_{m n}=\left\langle\Psi_{m} \mid \partial_{t} \Psi_{n}\right\rangle
$$

When computed by finite differences, time-derivative nonadiabatic couplings (TDNC) $\sigma_{m n}$ can be conveniently written in terms of wave function overlaps between consecutive time steps. Then, as proposed by Hammes-Schiffer and Tully [45], TDNC can be used to evaluate the fewest-switches probability formula, by directly replacing the inner product between the nonadiabatic coupling vector and the nuclear velocities, $\sigma_{m n}=\mathbf{F}_{m n} \cdot \mathbf{v}$. This procedure has become popular, as it allows us to overcome the cumbersome evaluation of nonadiabatic coupling vectors [46-48].

In the present work, TDNC are obtained by evaluating Equation (1) with the OD (for Orbital Derivative) method proposed in ref. [49]. This method requires computation of time derivatives (and wave function overlaps) on a basis of molecular orbitals, rather than on a basis of Slater determinants as usually done. (This latter approach will be referred as the DD (for Determinant Derivative) method).

The OD method is discussed in detail in ref. [49]. Here, we briefly outline the main points to explain its current implementation in NEWTON-X. Considering a configuration interaction expansion of singly excited determinants (CIS) $\left|\Phi_{i}^{a}\right\rangle=\hat{a}_{a}^{+} \hat{a}_{i}\left|\Phi_{0}\right\rangle$, the electronic wave function for state $m$ is:

$$
\left|\Psi_{m}\right\rangle=\sum_{i a} C_{i a}^{m}\left|\Phi_{i}^{a}\right\rangle
$$

The couplings between the excited states $m$ and $n$ can be evaluated as

$$
\sigma_{m n}=\sum_{i a} C_{i a}^{m} \partial_{t} C_{i a}^{n}+\sum_{i a b} C_{i a}^{m} C_{j b}^{n}\left\langle\varphi_{a} \mid \partial_{t} \varphi_{b}\right\rangle-\sum_{i j a} P_{i j} C_{i a}^{m} C_{j b}^{n}\left\langle\varphi_{j} \mid \partial_{t} \varphi_{i}\right\rangle,
$$

where $P_{i j}$ is a phase that depends on the ordering convention adopted for the molecular orbitals $\left\{\varphi_{k}\right\}$ in the Slater determinants.

Considering the overlap matrix between molecular orbitals from two consecutive time steps, the time derivatives of the molecular orbitals are evaluated by finite differences:

$$
\left\langle\varphi_{j} \mid \partial_{t} \varphi_{i}\right\rangle \approx \frac{\left\langle\varphi_{j}(t) \mid \varphi_{i}(t+\Delta t)\right\rangle}{\Delta t} \equiv \frac{S_{j i}(t, t+\Delta t)}{\Delta t}
$$

where $S_{j i}$ is the orbital overlap matrix. An orbital phase matching algorithm is used to assure the continuity of orbitals at different time steps.

The formal scaling of the TDNC evaluation is reduced from $N_{o c c}^{5} N_{v i r t}^{2}$ in the DD approach to $N_{o c c} N_{v i r t}^{2}$ in the OD. This method has had excellent results in comparison to the DD at significantly lower computational costs [49]. In the present simulations of thymine, for instance, computation of TDNC with the OD method was ten times faster than with the DD method.

We have implemented the OD method in NEWTON-X, where it is available for interfaces with GAUSSIAN [50] (CIS, TDA, and TDDFT methods) and TURBOMOLE (TDA, TDDFT, CC2, and ADC(2) methods). In particular, for the density functional based methods, approximated CIS wave functions are built using the Casida ansatz [51,52]. In the case of $\mathrm{ADC}(2)$ and CC2, approximated CIS wave functions are expressed in terms of Jacobian eigenvectors, where double excitations are neglected and the resulting wave functions are reorthonormalized [53].

Supplementary Materials: The following are available online at: http://www.mdpi.com/1420-3049/21/11/1603/s1, kinetic model to fit occupations and Cartesian coordinates for all structures.

Acknowledgments: Ljiljana Stojanović, Shuming Bai, and Mario Barbatti thank the support of the Aix-Marseille Initiative d'Excellence (A*MIDEX) grant (No. ANR-11-IDEX-0001-02) funded by the French Government 
"Investissements d'Avenir" program supervised by the Agence Nationale de la Recherche. This work was granted access to the HPC resources of Aix-Marseille Université financed by the project Equip@Meso (ANR-10-EQPX-29-01) also within the "Investissements d'Avenir" program. Artur F. Izmaylov acknowledges funding from a Sloan Research Fellowship and the Natural Sciences and Engineering Research Council of Canada (NSERC) through the Discovery Grants Program.

Author Contributions: M.B. and H.L. conceived and designed the simulations; L.S. and S.B. performed the simulations; J.N., R.C.-O., and A.F.I. developed and implemented the coupling method; L.S., R.C.-O., and M.B. wrote the paper.

Conflicts of Interest: The authors declare no conflict of interest. The founding sponsors had no role in the design of the study; in the collection, analyses, or interpretation of data; in the writing of the manuscript, and in the decision to publish the results.

\section{References}

1. Yu, H.; Sanchez-Rodriguez, J.A.; Pollum, M.; Crespo-Hernandez, C.E.; Mai, S.; Marquetand, P.; Gonzalez, L.; Ullrich, S. Internal conversion and intersystem crossing pathways in uv excited, isolated uracils and their implications in prebiotic chemistry. Phys. Chem. Chem. Phys. 2016, 18, 20168-20176. [CrossRef] [PubMed]

2. Kang, H.; Lee, K.T.; Jung, B.; Ko, Y.J.; Kim, S.K. Intrinsic lifetimes of the excited state of DNA and RNA bases. J. Am. Chem. Soc. 2002, 124, 12958-12959. [CrossRef] [PubMed]

3. Ullrich, S.; Schultz, T.; Zgierski, M.Z.; Stolow, A. Electronic relaxation dynamics in DNA and RNA bases studied by time-resolved photoelectron spectroscopy. Phys. Chem. Chem. Phys. 2004, 6, 2796-2801. [CrossRef]

4. McFarland, B.K.; Farrell, J.P.; Miyabe, S.; Tarantelli, F.; Aguilar, A.; Berrah, N.; Bostedt, C.; Bozek, J.D.; Bucksbaum, P.H.; Castagna, J.C.; et al. Ultrafast X-ray auger probing of photoexcited molecular dynamics. Nat. Commun. 2014, 5, 4235. [CrossRef] [PubMed]

5. Gonzalez-Vazquez, J.; Gonzalez, L.; Samoylova, E.; Schultz, T. Thymine relaxation after uv irradiation: The role of tautomerization and $\pi \sigma^{*}$ states. Phys. Chem. Chem. Phys. 2009, 11, 3927-3934. [CrossRef] [PubMed]

6. Gador, N.; Samoylova, E.; Smith, V.R.; Stolow, A.; Rayner, D.M.; Radloff, W.; Hertel, I.V.; Schultz, T. Electronic structure of adenine and thymine base pairs studied by femtosecond electron-ion coincidence spectroscopy. J. Phys. Chem. A 2007, 111, 11743-11749. [CrossRef] [PubMed]

7. Canuel, C.; Mons, M.; Piuzzi, F.; Tardivel, B.; Dimicoli, I.; Elhanine, M. Excited states dynamics of DNA and RNA bases: Characterization of a stepwise deactivation pathway in the gas phase. J. Chem. Phys. 2005, 122, 074316. [CrossRef] [PubMed]

8. Samoylova, E.; Schultz, T.; Hertel, I.V.; Radloff, W. Analysis of ultrafast relaxation in photoexcited DNA base pairs of adenine and thymine. Chem. Phys. 2008, 347, 376-382. [CrossRef]

9. Ligare, M.; Siouri, F.; Bludsky, O.; Nachtigallova, D.; de Vries, M.S. Characterizing the dark state in thymine and uracil by double resonant spectroscopy and quantum computation. Phys. Chem. Chem. Phys. 2015, 17, 24336-24341. [CrossRef] [PubMed]

10. Samoylova, E.; Lippert, H.; Ullrich, S.; Hertel, I.V.; Radloff, W.; Schultz, T. Dynamics of photoinduced processes in adenine and thymine base pairs. J. Am. Chem. Soc. 2005, 127, 1782-1786. [CrossRef] [PubMed]

11. Barbatti, M.; Borin, A.; Ullrich, S. Photoinduced processes in nucleic acids. In Photoinduced Phenomena in Nucleic Acids I; Barbatti, M., Borin, A.C., Ullrich, S., Eds.; Springer International Publishing: Cham, Switzerland, 2015; Volume 355, pp. 1-32.

12. Yamazaki, S.; Taketsugu, T. Nonradiative deactivation mechanisms of uracil, thymine, and 5-fluorouracil: A comparative ab initio study. J. Phys. Chem. A 2012, 116, 491-503. [CrossRef] [PubMed]

13. Perun, S.; Sobolewski, A.L.; Domcke, W. Conical intersections in thymine. J. Phys. Chem. A 2006, 110, 13238-13244. [CrossRef] [PubMed]

14. Szymczak, J.J.; Barbatti, M.; Soo Hoo, J.T.; Adkins, J.A.; Windus, T.L.; Nachtigallová, D.; Lischka, H. Photodynamics simulations of thymine: Relaxation into the first excited singlet state. J. Phys. Chem. A 2009, 113, 12686-12693. [CrossRef] [PubMed]

15. Zechmann, G.; Barbatti, M. Photophysics and deactivation pathways of thymine. J. Phys. Chem. A 2008, 112, 8273-8279. [CrossRef] [PubMed]

16. Serrano-Pérez, J.J.; González-Luque, R.; Merchán, M.; Serrano-Andrés, L. On the intrinsic population of the lowest triplet state of thymine. J. Phys. Chem. B 2007, 111, 11880-11883. [CrossRef] [PubMed] 
17. Bai, S.; Barbatti, M. Why replacing different oxygens of thymine with sulfur causes distinct absorption and intersystem crossing. J. Phys. Chem. A 2016. [CrossRef] [PubMed]

18. Merchán, M.; González-Luque, R.; Climent, T.; Serrano-Andrés, L.; Rodriuguez, E.; Reguero, M.; Pelaez, D. Unified model for the ultrafast decay of pyrimidine nucleobases. J. Phys. Chem. B 2006, 110, 26471-26476. [CrossRef] [PubMed]

19. Asturiol, D.; Lasorne, B.; Robb, M.A.; Blancafort, L. Photophysics of the $\pi, \pi^{*}$ and $n, \pi^{*}$ states of thymine: Ms-CASPT2 minimum-energy paths and CASSCF on-the-fly dynamics. J. Phys. Chem. A 2009, 113, 10211-10218. [CrossRef] [PubMed]

20. Lan, Z.; Fabiano, E.; Thiel, W. Photoinduced nonadiabatic dynamics of pyrimidine nucleobases: On-the-fly surface-hopping study with semiempirical methods. J. Phys. Chem. B 2009, 113, 3548-3555. [CrossRef] [PubMed]

21. González, L.; González-Vázquez, J.; Samoylova, E.; Schultz, T. On the puzzling deactivation mechanism of thymine after light irradiation. AIP Conf. Proc. 2008, 1080, 169-175.

22. Arbelo-González, W.; Crespo-Otero, R.; Barbatti, M. Steady and time-resolved photoelectron spectra based on nuclear ensembles. J. Chem. Theory Comput. 2016, 12, 5037-5049. [CrossRef] [PubMed]

23. Hudock, H.R.; Levine, B.G.; Thompson, A.L.; Satzger, H.; Townsend, D.; Gador, N.; Ullrich, S.; Stolow, A.; Martínez, T.J. Ab initio molecular dynamics and time-resolved photoelectron spectroscopy of electronically excited uracil and thymine. J. Phys. Chem. A 2007, 111, 8500-8508. [CrossRef] [PubMed]

24. Barbatti, M.; Aquino, A.J.A.; Szymczak, J.J.; Nachtigallová, D.; Hobza, P.; Lischka, H. Relaxation mechanisms of UV-photoexcited DNA and RNA nucleobases. Proc. Natl. Acad. Sci. USA 2010, 107, 21453-21458. [CrossRef] [PubMed]

25. Picconi, D.; Barone, V.; Lami, A.; Santoro, F.; Improta, R. The interplay between $\pi \pi^{*} / \mathrm{n} \pi^{*}$ excited states in gas-phase thymine: A quantum dynamical study. ChemPhysChem 2011, 12, 1957-1968. [CrossRef] [PubMed]

26. Cremer, D.; Pople, J.A. General definition of ring puckering coordinates. J. Am. Chem. Soc. 1975, 97, 1354-1358. [CrossRef]

27. Zhu, X.-M.; Wang, H.-G.; Zheng, X.; Phillips, D.L. Role of ribose in the initial excited state structural dynamics of thymidine in water solution: A resonance raman and density functional theory investigation. J. Phys. Chem. B 2008, 112, 15828-15836. [CrossRef] [PubMed]

28. Abouaf, R.; Pommier, J.; Dunet, H. Electronic and vibrational excitation in gas phase thymine and 5-bromouracil by electron impact. Chem. Phys. Lett. 2003, 381, 486-494. [CrossRef]

29. Tuna, D.; Lefrancois, D.; Wolański, Ł.; Gozem, S.; Schapiro, I.; Andruniów, T.; Dreuw, A.; Olivucci, M. Assessment of approximate coupled-cluster and algebraic-diagrammatic-construction methods for groundand excited-state reaction paths and the conical-intersection seam of a retinal-chromophore model. J. Chem. Theory Comput. 2015, 11, 5758-5781. [CrossRef] [PubMed]

30. Trofimov, A.B.; Schirmer, J. An efficient polarization propagator approach to valence electron excitation spectra. J. Phys. B At. Mol. Opt. Phys. 1995, 28, 2299-2324. [CrossRef]

31. Schirmer, J. Beyond the random-phase approximation: A new approximation scheme for the polarization propagator. Phys. Rev. A 1982, 26, 2395-2416. [CrossRef]

32. Dunning, T.H., Jr. Gaussian basis sets for use in correlated molecular calculations. I. The atoms boron through neon and hydrogen. J. Chem. Phys. 1989, 90, 1007-1023. [CrossRef]

33. Fogarasi, G.; Zhou, X.F.; Taylor, P.W.; Pulay, P. The calculation of abinitio molecular geometries-Efficient optimization by natural internal coordinates and empirical correction by offset forces. J. Am. Chem. Soc. 1992, 114, 8191-8201. [CrossRef]

34. Crespo-Otero, R.; Barbatti, M. Spectrum simulation and decomposition with nuclear ensemble: Formal derivation and application to benzene, furan and 2-phenylfuran. Theor. Chem. Acc. 2012, 131, 1237. [CrossRef]

35. Tully, J.C. Molecular-dynamics with electronic-transitions. J. Chem. Phys. 1990, 93, 1061-1071. [CrossRef]

36. Granucci, G.; Persico, M. Critical appraisal of the fewest switches algorithm for surface hopping. J. Chem. Phys. 2007, 126, 134114. [CrossRef] [PubMed]

37. Swope, W.C.; Andersen, H.C.; Berens, P.H.; Wilson, K.R. A computer-simulation method for the calculation of equilibrium-constants for the formation of physical clusters of molecules-Application to small water clusters. J. Chem. Phys. 1982, 76, 637-649. [CrossRef]

38. Butcher, J. A modified multistep method for the numerical integration of ordinary differential equations. J. Assoc. Comput. Mach. 1965, 12, 124-135. [CrossRef] 
39. Boeyens, J.C.A. The conformation of six-membered rings. J. Chem. Crystallogr. 1978, 8, 317-320. [CrossRef]

40. Ahlrichs, R.; Bär, M.; Häser, M.; Horn, H.; Kölmel, C. Electronic-structure calculations on workstation computers-The program system turbomole. Chem. Phys. Lett. 1989, 162, 165-169. [CrossRef]

41. Barbatti, M.; Ruckenbauer, M.; Plasser, F.; Pittner, J.; Granucci, G.; Persico, M.; Lischka, H. Newton-X: A surface-hopping program for nonadiabatic molecular dynamics. WIREs Comput. Mol. Sci. 2014, 4, $26-33$. [CrossRef]

42. Barbatti, M.; Granucci, G.; Ruckenbauer, M.; Plasser, F.; Crespo-Otero, R.; Pittner, J.; Persico, M.; Lischka, H. Newton-X: A Package for Newtonian Dynamics Close to the Crossing Seam. 2013. Available online: http:/ / www.Newtonx.Org (accessed on 1 September 2016).

43. Levine, B.G.; Coe, J.D.; Martínez, T.J. Optimizing conical intersections without derivative coupling vectors: Application to multistate multireference second-order perturbation theory (MS-CASPT2). J. Phys. Chem. B 2008, 112, 405-413. [CrossRef] [PubMed]

44. Spek, A.L. Single-crystal structure validation with the program platon. J. Appl. Crystallogr. 2003, 36, 7-13. [CrossRef]

45. Hammes-Schiffer, S.; Tully, J.C. Proton-transfer in solution-Molecular-dynamics with quantum transitions. J. Chem. Phys. 1994, 101, 4657-4667. [CrossRef]

46. Pittner, J.; Lischka, H.; Barbatti, M. Optimization of mixed quantum-classical dynamics: Time-derivative coupling terms and selected couplings. Chem. Phys. 2009, 356, 147-152. [CrossRef]

47. Werner, U.; Mitrić, R.; Suzuki, T.; Bonačić-Koutecký, V. Nonadiabatic dynamics within the time dependent density functional theory: Ultrafast photodynamics in pyrazine. Chem. Phys. 2008, 349, 319-324. [CrossRef]

48. Tapavicza, E.; Tavernelli, I.; Rothlisberger, U. Trajectory surface hopping within linear response time-dependent density-functional theory. Phys. Rev. Lett. 2007, 98, 023001-023004. [CrossRef] [PubMed]

49. Ryabinkin, I.G.; Nagesh, J.; Izmaylov, A.F. Fast numerical evaluation of time-derivative nonadiabatic couplings for mixed quantum-classical methods. J. Phys. Chem. Lett. 2015, 6, 4200-4203. [CrossRef] [PubMed]

50. Frisch, M.J.; Trucks, G.W.; Schlegel, H.B.; Scuseria, G.E.; Robb, M.A.; Cheeseman, J.R.; Scalmani, G.; Barone, V.; Mennucci, B.; Petersson, G.A.; et al. Gaussian 09, Revision D.01; Gaussian, Inc.: Wallingford, CT, USA, 2013.

51. Casida, M. Time-dependent density functional response theory for molecules. In Recent Advances in Density Functional Methods, Part I; Chong, D., Ed.; World Scientific: Singapore, 1995; pp. 155-192.

52. Barbatti, M.; Crespo-Otero, R. Surface hopping dynamics with dft excited states. In Density-Functional Methods for Excited States; Ferré, N., Filatov, M., Huix-Rotllant, M., Eds.; Springer International Publishing: Cham, Switzerland, 2016; pp. 415-444.

53. Plasser, F.; Crespo-Otero, R.; Pederzoli, M.; Pittner, J.; Lischka, H.; Barbatti, M. Surface hopping dynamics with correlated single-reference methods: $9 H$-adenine as a case study. J. Chem. Theory Comput. 2014, 10, 1395-1405. [CrossRef] [PubMed]

(C) 2016 by the authors; licensee MDPI, Basel, Switzerland. This article is an open access article distributed under the terms and conditions of the Creative Commons Attribution (CC-BY) license (http:/ / creativecommons.org/licenses/by/4.0/). 